\title{
Evaluation of the banana cultivars Zelig, Grande Naine and Gruesa under different environmental conditions in the Canary Islands
}

Juan Cabrera Cabrera, Victor Galán Saúco*

Instituto Canario de Investigaciones Agrarias, Departamento de Fruticultura Tropical, Apartado 60, 38200 La Laguna, Tenerife, Islas Canarias, Spain vgalan@icia.es
* Correspondence and reprints

Received 7 March 2005 Accepted 2 September 2005

Fruits, 2005, vol. 60, p. 357-369 (C) 2005 Cirad/EDP Sciences All rights reserved DOI: 10.1051/fruits:2005041

RESUMEN ESPAÑOL, p. 369

\section{Evaluation of the banana cultivars Zelig, Grande Naine and Gruesa under different environmental conditions in the Canary Islands.}

Abstract - Introduction. A study was done to compare Gruesa, a local dwarf Cavendish selection, with two other cultivars of the Cavendish subgroup in the open air or under greenhouse conditions in two localities of the Canary Islands. Materials and methods. The trials were set up with the cultivars Zelig, Grande Naine and Gruesa in four plots on the northern slope: one outdoors and three under three different greenhouse covers (one with polyethylene lamina and the other two with different types of mesh), and in one outdoor plot on the southern slope of the island of Tenerife. Morphological, phenological and production characteristics were studied over three productive cycles. Results. Significant differences were found in both plant height (Grande Naine $>$ Zelig $>$ Gruesa) and pseudostem thickness (Gruesa $>$ Grande Naine $=$ Zelig). Each cultivar showed a stable [height:thickness] ratio, remaining the same in all trial conditions. Gruesa cycle length was longer than those of the other two cultivars, emitting more leaves until bunch emergency. Gruesa produced the most hands per bunch and the most fingers per hand. In all cultivars, finger length was directly related to pseudostem height. All three cultivars produced well, with fruits very similar in organoleptic traits. A reduction in cycle length and a slight increase in productivity were observed in the northern plot under the polyethylene cover compared with the plots under other covers and outdoors. Conclusion. Gruesa appears more recommendable than Zelig and Grande Naine for open-air plantations in windy localities.

Spain / Canary Islands / Musa / variety trials / cropping systems / greenhouses / solar radiation / phenology

Évaluation des bananiers Zelig, Grande Naine et Gruesa dans différents environnements des îles Canaries.

Résumé - Introduction. Une étude a été faite pour comparer le cultivar Gruesa, une sélection locale de Cavendish nain, avec deux autres cultivars du sous-groupe Cavendish en plein air ou sous serre dans deux localités des îles Canaries. Matériel et méthodes. Des essais ont été mis en place avec les cultivars Zelig, Grande Naine et Gruesa sur quatre parcelles du versant nord de l'île de Tenerife : une parcelle en extérieur et trois parcelles sous trois serres présentant des couvertures différentes (une avec feuille de polyéthylène et les deux autres avec différents types de mailles), et sur une parcelle du versant méridional. Des caractéristiques morphologiques, phénologiques et de production ont été étudiées sur trois cycles de productions. Résultats. Des différences significatives ont été trouvées entre la hauteur des plants des différents cultivars (Grande Naine > Zelig > Gruesa) et le diamètre de leur pseudo-tige (Gruesa > Grande Naine $=$ Zelig). Chaque cultivar a eu un rapport [hauteur:diamètre] stable, celui-ci restant le même quelles que soient les conditions d'essai. Le cycle de production Gruesa a été plus long que celui des deux autres cultivars, ce bananier émettant davantage de feuilles jusqu'à l'émergence du régime. Gruesa a produit le plus de mains par régime et le plus de doigts par main. Pour tous les cultivars, la longueur des doigts a été directement liée à la taille des pseudo-tiges. Chacun des trois cultivars a donné une bonne production, avec des fruits présentant des caractéristiques organoleptiques très semblables. Il y a eu une réduction de la longueur de cycle et une légère augmentation de la productivité de la parcelle implantée sous couverture de polyéthylène au nord de l'île, comparée aux deux parcelles mises sous les autres couvertures et à la parcelle extérieure. Conclusion. Le cultivar Gruesa semble plus adapté que Zelig et Grande Naine à des plantations de plein air soumises aux vents.

Espagne / Canaries (îles) / Musa / essai de variété / système de culture / serre / radiation solaire / phénologie 


\section{Introduction}

The Canary Islands had 9614 ha of banana plantations in $2002^{1}$, with approximately 3000 ha under greenhouse production. The cultivar distribution is approximately 4500 ha of Dwarf Cavendish, 3000 ha of Grande Naine, and 2000 ha of Gruesa, with assorted Cavendish subgroup cultivars making up the rest.

Gruesa, a local banana Dwarf Cavendish selection, is presently the preferred cultivar for new plantations in the region, substituting Grande Naine as the choice cultivar [1]. Gruesa is probably a natural mutation of Dwarf Cavendish and was first observed in several plantations on the island of $\mathrm{La}$ Palma. Besides high yield, the popularity of this selection is mainly due to its similarity to Dwarf Cavendish regarding short stature and finger length: this last characteristic is particularly attractive to growers who want to promote a market clearly differentiated from the larger fruit typically produced by the multinational brands, without renouncing the high yields of Grande Naine [2, 3].

Grande Naine and, to a lesser extent, Zelig, an Israeli selection, have proven their worth in terms of productivity [2, 3], but plant height can be a problem, especially after the second year of planting, particularly in open-air plots. On the other hand, Gruesa is somewhat shorter, making cultural practices easier and affording more protection against wind damage.

Our trial was set to compare the potential of the three cultivars Grande Naine, Gruea and Zelig during three cycles (plant crop and first and second ratoons) under the different environmental conditions in which bananas are cultivated in the Canary Islands: in the open air on the southern, warmer slope, and in greenhouses on the northern, cooler side of the island of Tenerife.

\section{Materials and methods}

In August, 1997, trials were set up in five plots belonging to the Instituto Canario de

\footnotetext{
${ }^{1}$ According to www.gobiernodecanarias.org.
}

Investigaciones Agrarias (ICIA) on the island of Tenerife, using in vitro plants of the cultivars Grande Naine, Gruesa and Zelig, produced by a local tissue culture laboratory (CULTESA).

The first plot was an open-air plot, located in Guia de Isora (Finca Cueva del Polvo) at an altitude slightly below $100 \mathrm{~m}$, on the southern and warmest slope of the island. The experimental design was random blocks with four replications of 72 plants per cultivar and 20 useful plants per cultivar.

Four other plots were located on the northern and cooler slope in Valle Guerra (Finca Pajalillos) at an altitude slightly below $100 \mathrm{~m}$. The experimental design was random blocks without replications, with three greenhouse plots ( 56 plants per cultivar in each greenhouse) and one open-air plot (96 plants per cultivar). Each of these three greenhouses had a different covering material: 720-gauge polyethylene on all sides and the roof (PE); mixed monofilament/raffia mesh on the sides and roof (MM); and 6-by-6 monofilament mesh for the roof and monofilament/raffia-mix mesh for sides $($ M $6 \times 6)$. Each greenhouse covers $1000 \mathrm{~m}^{2}$ and was originally designed for ICIA trials on different cladding materials [4].

In all treatments, the planting density was 2400 plants per ha. Two plants were planted per hole, with $1.67 \mathrm{~m}$ between holes and $5.0 \mathrm{~m}$ between rows. Drip irrigation with fertigation was scheduled. Each cultivar received its standard crop management.

The main parameters recorded were leaf emission rate; total number of leaves emitted until bunch emergence (for the first cycle, leaves greater than $25 \mathrm{~cm}$ in width); leaves emitted after the orthogonal leaf (second and third cycles); pseudostem height, measured from the soil to the point of the emerging bunch; pseudostem width at $1 \mathrm{~m}$ from the soil; date of bunch emergence; number of hands per bunch; harvest date; bunch weight; length and calibre of the characteristic fruit of the second top and bottom hands; and number of fruits of the second top and bottom hands produced during the second and third cycles. The harvesting point was determined according to commercial practice when fruit had filled out 

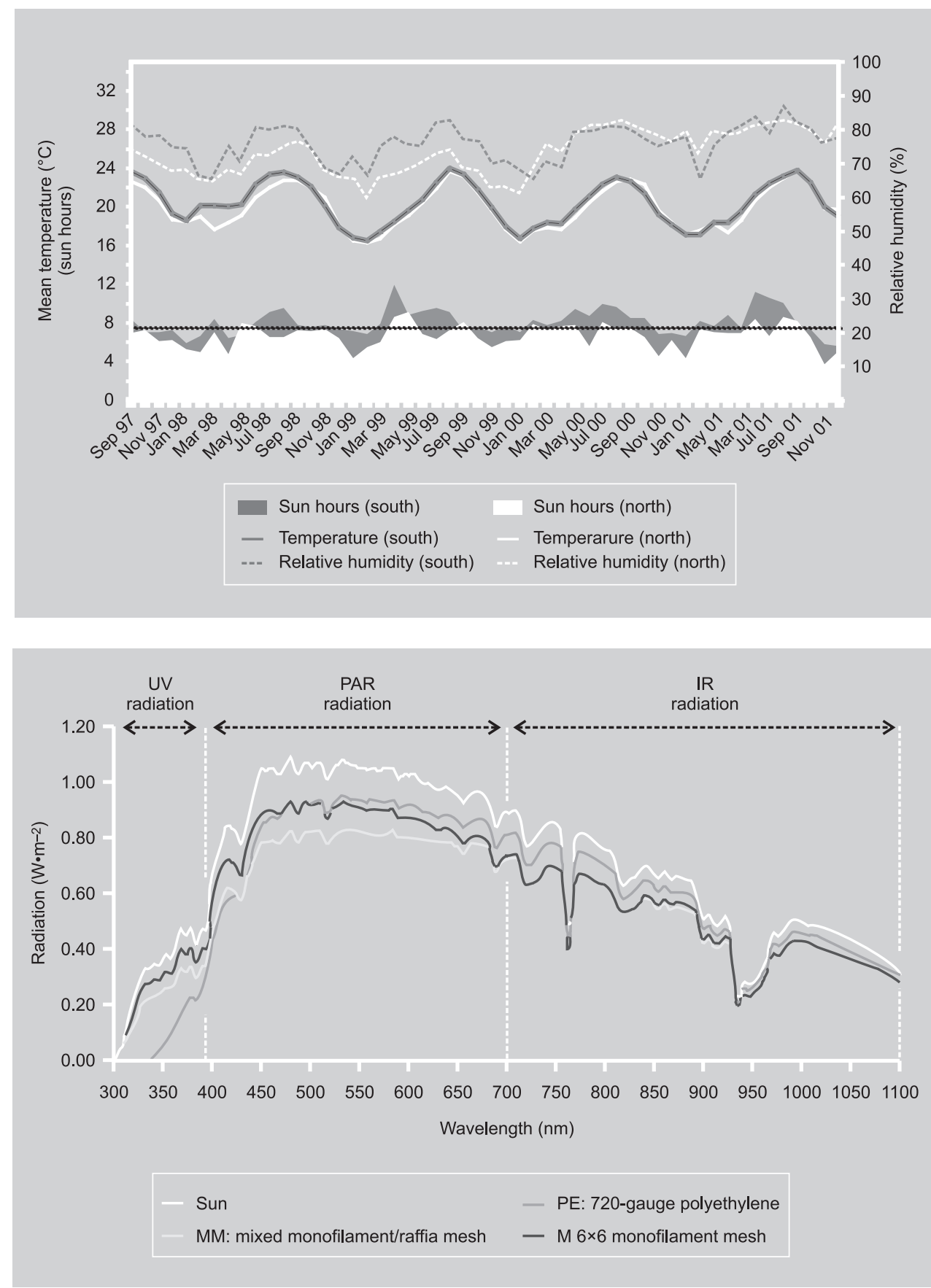

enough to guarantee eventual prime commercial category.

Data were subject to an analysis of variance and means were compared by using Tukey's test.

\section{Figure 1.}

Mean monthly climatic data for Pajalillos, Valle Guerra, northern Tenerife (means for total period studied: $20.0^{\circ} \mathrm{C}$, $75.9 \% \mathrm{RH},: 6.7$ sun hours) and Cueva del Polvo, southern Tenerife (means for total period studied: $20.6{ }^{\circ} \mathrm{C}, 73.4 \% \mathrm{RH}$, 8.1 sun hours) in the Canary Islands, Spain.

\section{Figure 2.}

Radiation spectrum for three different greenhouse covers in Pajalillos, Valle Guerra, northern Tenerife, 2 January 2000, 12:30 PM (Canary Islands, Spain). 
Table I.

Comparison of radiation data for different wavelengths measured for three different greenhouse covers (PE: 720-gauge polyethylene on all sides and roof; MM: mixed monofilament/raffia mesh on sides and roof; M 6 × 6: 6-by-6 monofilament mesh for roof and monofilament / raffia-mix mesh for sides) in Pajalillos, Valle Guerra, northern Tenerife, 2 January 2000, 12:30 PM (Canary Islands, Spain).

\begin{tabular}{|c|c|c|}
\hline Treatment & $W \cdot m^{-2}$ & $\begin{array}{c}\text { Radiation \% } \\
\text { compared with the sun }\end{array}$ \\
\hline \multicolumn{3}{|c|}{ UV radiation between (300 and 400$) \mathrm{nm}$} \\
\hline Sun & 33 & 100 \\
\hline $\mathrm{PE}$ & 9 & 28 \\
\hline$M 6 \times 6$ & 28 & 84 \\
\hline MM & 23 & 69 \\
\hline \multicolumn{3}{|c|}{$P A R$ radiation between $(400$ and 700$) \mathrm{nm}$} \\
\hline Sun & 295 & 100 \\
\hline PE & 254 & 86 \\
\hline$M 6 \times 6$ & 252 & 85 \\
\hline MM & 229 & 78 \\
\hline \multicolumn{3}{|c|}{ IR radiation between (700 and 1100$) \mathrm{nm}$} \\
\hline Sun & 230 & 100 \\
\hline $\mathrm{PE}$ & 212 & 92 \\
\hline M $6 \times 6$ & 193 & 84 \\
\hline MM & 191 & 83 \\
\hline \multicolumn{3}{|c|}{ Total radiation between (300 and 1100) $\mathrm{nm}$} \\
\hline Sun & 558 & 100 \\
\hline PE & 476 & 85 \\
\hline M $6 \times 6$ & 473 & 85 \\
\hline MM & 442 & 79 \\
\hline
\end{tabular}

mean temperatures registered inside each greenhouse as well as outdoors for the northern trial over one crop cycle (figure 3).

Severe storms in January, 1999, with winds of over $100 \mathrm{~km} \cdot \mathrm{h}^{-1}$, caused considerable damage in the open-air plot of the northern trial, but greenhouse plots were not affected; no data after first cycle bunch emergence could thus be recorded in this open-air plot.

\section{Results}

\subsection{Morphological characteristics}

For all plots in both localities, significant differences in pseudostem height were found for all three cultivars and in all cycles, with
Grande Naine being the tallest and Gruesa the shortest (table II). Gruesa's pseudostem width was always significantly greater than that of the other two cultivars, except for the Gruesa and Grande Naine in the second and third cycles in the northern locality; differences between Grande Naine and Zelig were practically nonexistent and, when they did occur, they were indistinct, varying in relation to cycle rather than to cultivar.

The differences in the ratio [height / width] of the pseudostem were always significant for all cycles and plots, with Grande Naine $>$ Zelig $>$ Gruesa.

Regarding plots of the northern locality, the greenhouse with MM structure always produced the tallest plants, although height was only significantly inferior for the first cycle. As mentioned above, data for the outdoor northern locality were restricted to the first cycle, but such data as was recorded showed that these plants were significantly shorter than those growing in any of the greenhouses. Pseudostem width for greenhouse plants was consistently greater than that of either of the open-air plots, and no significant differences were found between the greenhouse plants with the exception of a greater width for PE structure plants during the first cycle. No significant differences in height and width were found, either among the greenhouse plots or between these and the outdoor plot.

Regarding the 1999 storm damage to the open-air plants of the northern locality, it was interesting to note that Gruesa plants showed better resistance, with only $7 \%$ of plants being toppled by the wind, while Zelig lost 38\% and Grande Naine 53\% of the plants: this appears to be proportionate to height and width (data not shown). Neither the plants nor the structures of the greenhouse plot were affected by the storm.

\subsection{Phenological characteristics}

In the southern locality, leaf emission rate was slightly superior in Gruesa, showing significant differences compared with Grande Naine and Zelig in the second cycle and between Gruesa and Zelig for the third cycle (table III). This was not appreciable for the 
greenhouse plots of the northern locality, although the leaf emission rate was always greater for Gruesa than for Grande Naine. No significant differences between Zelig and Grande Naine were detected in any treatment.

Although not always significant, there were clear differences in favour of all cultivars growing in the PE greenhouse structure compared with the other two greenhouse coverings, and all plants growing under any of the greenhouses showed better phenological development than those growing in the open-air plot of the northern trial plots, at least for the only cycle for which data were available.

The total number of leaves emitted both after leaves were greater than $25 \mathrm{~cm}$ in width (first cycle) as well as after the orthogonal leaf (second and third cycles) showed that Gruesa was always significantly higher compared with Grande Naine and Zelig, but only in the first cycle in the southern locality. Significant differences were also detected between Zelig (greater number of leaves emitted) and Grande Naine. Total number of leaves was also significantly higher for the outdoor plot versus all coverings, but differences between covers, although significant, were rather inconsistent, varying from cycle to cycle.

Gruesa always bunched significantly later than Grande Naine and Zelig in the southern locality, with the same tendency also observed in the second cycle in the greenhouse plots of the northern locality. The earliest date of emergence occurred in the PE greenhouse structure, with significant differences in the first and second cycles compared with the M $6 \times 6$ greenhouse structure, and with the MM greenhouse structure in the third cycle; no differences in any cycle occurred between these latter two treatments. In the only cycle for which data could be recorded comparing the greenhouse plots with the open-air plot of the northern locality, emergence dates in the open air were significantly later than those of any of the greenhouse plots.

In the southern locality, the interval between bunch emergence and harvest was also significantly longer for Gruesa com-

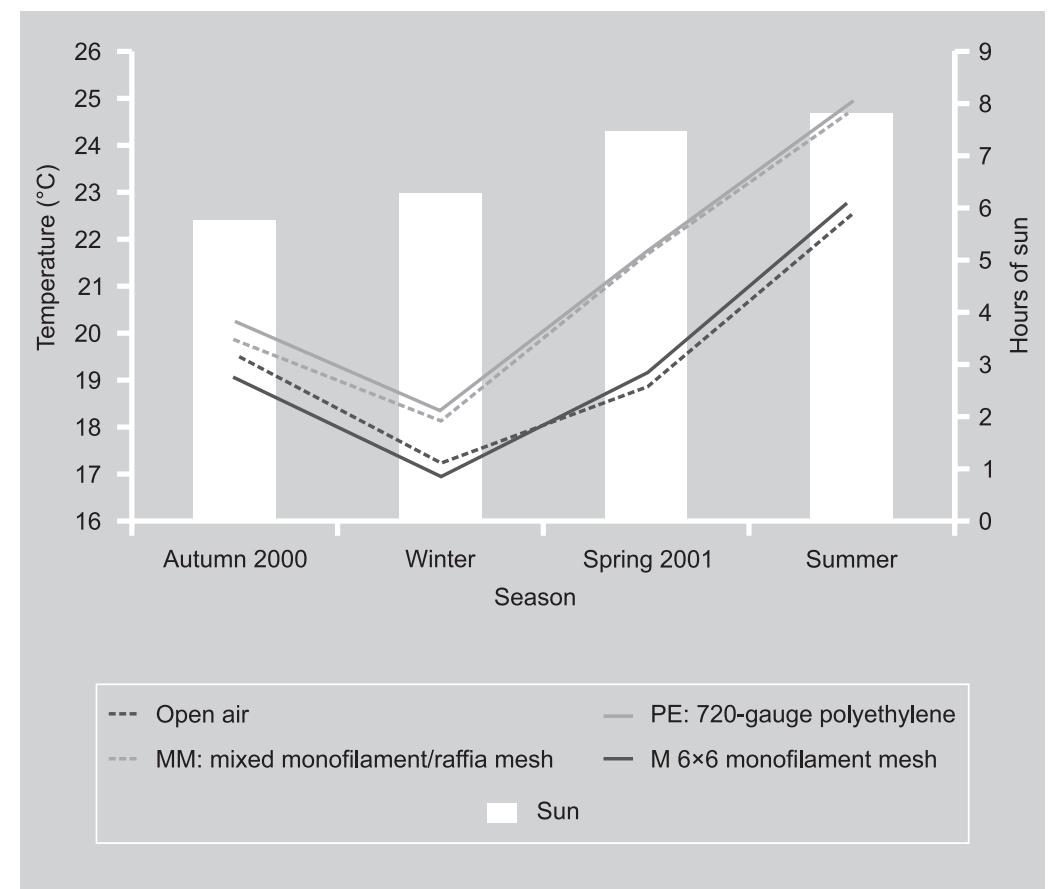

pared with the other two cultivars; Grande Naine and Zelig showed no differences between them. In the northern trial plots, no significant differences between cultivars were seen for the greenhouse plots. However, the claddings did have significant effects, shortening the emergence-harvest interval in favour of the PE greenhouse structure, followed by the MM structure and, finally, the M $6 \times 6$ structure, with significant differences in the first cycle, none in the second, and again $\mathrm{PE}<\mathrm{MM}<\mathrm{M} 6 \times 6$ in the third cycle.

Significant differences were found between the three cultivars during the first cycle in the southern trial for the total cycle length, with Grande Naine ready for harvest almost 1 month earlier than Gruesa, with Zelig intermediate in the first cycle of the trial. These differences continued in the successive cycles, but Grande Naine and Zelig became virtually the same for this parameter. Results in the northern trial followed the same pattern; although the differences were not significant, Gruesa did average a longer interval than the other cultivars. With regard specifically to greenhouse influence on planting date to harvest, the PE greenhouse
Figure 3.

Mean temperatures recorded in the open-air plot and under three different greenhouse covers in northern Tenerife, Valle Guerra (Canary Islands, Spain). 
Table II.

Morphological characteristics of banana cvs. Grande Naine, Gruesa and Zelig, grown in the south and the north of Tenerife (Canary Islands, Spain).

(a) Cultivar comparison in southern trial plots.

\begin{tabular}{lcccc} 
Cycle & Cultivar & \multicolumn{3}{c}{ Pseudostem } \\
& & $\begin{array}{c}\text { Height } \\
(\mathrm{cm})\end{array}$ & $\begin{array}{c}\text { Width } \\
(\mathrm{mm})\end{array}$ & Ratio \\
\hline Parent crop & Grande Naine & $310.8 \mathrm{a}$ & $77.8 \mathrm{~b}$ & $4.0 \mathrm{a}$ \\
& Gruesa & $254.2 \mathrm{c}$ & $87.4 \mathrm{a}$ & $2.9 \mathrm{c}$ \\
1st ratoon crop & Zelig & $286.2 \mathrm{~b}$ & $79.2 \mathrm{~b}$ & $3.6 \mathrm{~b}$ \\
& Grande Naine & $355.1 \mathrm{a}$ & $91.3 \mathrm{~b}$ & $3.9 \mathrm{a}$ \\
& Gruesa & $281.4 \mathrm{c}$ & $101.7 \mathrm{a}$ & $2.8 \mathrm{c}$ \\
2nd ratoon crop & Zelig & $317.8 \mathrm{~b}$ & $89.7 \mathrm{~b}$ & $3.5 \mathrm{~b}$ \\
& Grande Naine & $345.0 \mathrm{a}$ & $90.8 \mathrm{~b}$ & $3.8 \mathrm{a}$ \\
& Gruesa & $268.2 \mathrm{c}$ & $100.4 \mathrm{a}$ & $2.7 \mathrm{c}$ \\
& Zelig & $305.5 \mathrm{~b}$ & $89.5 \mathrm{~b}$ & $3.4 \mathrm{~b}$
\end{tabular}

(b) Cultivar comparison in northern trial plots.

\begin{tabular}{lcccc} 
Cycle & Cultivar & \multicolumn{3}{c}{ Pseudostem } \\
& & $\begin{array}{c}\text { Height } \\
(\mathrm{cm})\end{array}$ & $\begin{array}{c}\text { Width } \\
(\mathrm{mm})\end{array}$ & Ratio \\
\hline \multirow{2}{*}{ Parent crop } & Grande Naine & $283.0 \mathrm{a}$ & $70.0 \mathrm{~b}$ & $4.0 \mathrm{a}$ \\
& Gruesa & $218.0 \mathrm{c}$ & $78.0 \mathrm{a}$ & $2.8 \mathrm{c}$ \\
\multirow{3}{*}{ 1st ratoon crop } & Zelig & $254.0 \mathrm{~b}$ & $71.0 \mathrm{~b}$ & $3.6 \mathrm{~b}$ \\
& Grande Naine & $309.6 \mathrm{a}$ & $81.8 \mathrm{ab}$ & $3.8 \mathrm{a}$ \\
& Gruesa & $225.7 \mathrm{c}$ & $85.0 \mathrm{a}$ & $2.7 \mathrm{c}$ \\
2nd ratoon crop & Zelig & $268.4 \mathrm{~b}$ & $79.0 \mathrm{~b}$ & $3.4 \mathrm{~b}$ \\
& Grande Naine & $330.4 \mathrm{a}$ & $85.2 \mathrm{ab}$ & $3.9 \mathrm{a}$ \\
& Gruesa & $248.2 \mathrm{c}$ & $90.8 \mathrm{a}$ & $2.7 \mathrm{c}$ \\
& Zelig & $293.0 \mathrm{~b}$ & $83.0 \mathrm{~b}$ & $3.5 \mathrm{~b}$
\end{tabular}

(c) Comparison of greenhouse covers (PE: 720-gauge polyethylene on all sides and roof; MM: mixed monofilament / raffia mesh on sides and roof; M $6 \times 6$ : 6-by-6 monofilament mesh for roof and monofilament / raffia-mix mesh for sides) in northern trial plots.

\begin{tabular}{|c|c|c|c|c|}
\hline \multirow[t]{2}{*}{ Cycle } & \multirow{2}{*}{$\begin{array}{l}\text { Greenhouse } \\
\text { cover }\end{array}$} & \multicolumn{3}{|c|}{ Pseudostem } \\
\hline & & $\begin{array}{l}\text { Height } \\
(\mathrm{cm})\end{array}$ & $\begin{array}{l}\text { Width } \\
(\mathrm{mm})\end{array}$ & Ratio \\
\hline \multirow[t]{4}{*}{ Parent crop } & PE & $272.0 \mathrm{a}$ & $79.0 \mathrm{a}$ & $3.5 \mathrm{a}$ \\
\hline & MM & $260.0 \mathrm{~b}$ & $77.0 \mathrm{~b}$ & $3.4 \mathrm{a}$ \\
\hline & M $6 \times 6$ & $270.0 \mathrm{a}$ & $77.0 \mathrm{~b}$ & $3.5 \mathrm{a}$ \\
\hline & Open-air ${ }^{1}$ & $203.0 \mathrm{c}$ & $61.0 \mathrm{c}$ & $3.4 \mathrm{a}$ \\
\hline \multirow[t]{3}{*}{ 1st ratoon crop } & PE & $268.0 \mathrm{a}$ & $82.0 \mathrm{a}$ & $3.3 \mathrm{a}$ \\
\hline & $\mathrm{MM}$ & $261.0 \mathrm{a}$ & $81.0 \mathrm{a}$ & $3.2 \mathrm{a}$ \\
\hline & M $6 \times 6$ & $275.0 \mathrm{a}$ & $83.0 \mathrm{a}$ & $3.3 \mathrm{a}$ \\
\hline \multirow[t]{3}{*}{ 2nd ratoon crop } & PE & $299.0 \mathrm{a}$ & $86.0 \mathrm{a}$ & $3.5 \mathrm{a}$ \\
\hline & MM & $279.0 \mathrm{a}$ & $85.0 \mathrm{a}$ & $3.3 \mathrm{a}$ \\
\hline & M $6 \times 6$ & $294.0 \mathrm{a}$ & $88.0 \mathrm{a}$ & $3.3 \mathrm{a}$ \\
\hline
\end{tabular}

Means followed by the same letters are not significantly different $(p<0.05)$, applying Tukey's test.

${ }^{1}$ Data only available for parent crop cycle (hands), due to storm damage. plot showed the shortest interval (significant in the first and third cycles), with differences between the MM structure and the M $6 \times 6$ structure - in favour of the MM structure in the first cycle only.

\subsection{Production characteristics}

None of the cultivars showed significant differences in bunch weight during the first two cycles of the southern trial, although Zelig was significantly different, by default, in the third cycle (table IV). However, there did appear to be a slight difference in favour of Grande Naine in that trial, which again appeared in the northern trial, where it became significant in the first and second cycles. Differences due to greenhouse cladding were not entirely clear although firstcycle weights were significantly greater in the PE greenhouse structure, but were similar between the PE and M $6 \times 6$ structures in the second cycle and, by the third cycle, no significant differences could be found among covers.

Means of the three cycles show high yields for all three cultivars, averaging $47 \mathrm{~kg}$ in the south and $43 \mathrm{~kg}$ in the north (slightly higher for the greenhouse plots compared with the open-air plot).

Yields for the southern locality were higher than those of the northern greenhouse treatments. In general, during the third cycle, Grande Naine and Gruesa produced more than Zelig in the southern trial, with Grande Naine producing the best of the three. Accumulating the yields for all three cycles, Grande Naine produced $7 \%$ more than Zelig and 3\% more than Gruesa. In the northern trial, Grande Naine was also the best in the first and second cycles, but in the third cycle, yields were very similar for all three cultivars with a slight increase noted for Gruesa. The accumulated northern yield showed Grande Naine producing 8\% more than Gruesa and 6\% more than Zelig. Regarding greenhouse influence on yield, the PE structure showed a $9 \%$ increase over the other greenhouse plots in terms of accumulated yields of all three cycles (on a cycleper-cycle basis, differences are not so appreciable). 
Table III.

Phenological characteristics of banana cvs. Grande Naine, Gruesa and Zelig grown in the south and the north of Tenerife (Canary Islands, Spain).

(a) Cultivar comparison in southern trial plots.

\begin{tabular}{|c|c|c|c|c|c|c|c|c|}
\hline \multirow[t]{2}{*}{ Cycle } & \multirow[t]{2}{*}{ Cultivar } & \multicolumn{2}{|c|}{ Leaves emitted $>25 \mathrm{~cm}$} & \multicolumn{2}{|c|}{ Bunch } & \multicolumn{3}{|c|}{ Intervals (days) from planting date } \\
\hline & & $\begin{array}{l}\text { See interval } 1,2 \text { or } 3 \\
\text { according to the cycle }\end{array}$ & $\begin{array}{l}\text { Total at } \\
\text { emergence }\end{array}$ & Emergence & Harvest & $\begin{array}{l}\text { Planting date- } \\
\text { emergence }\end{array}$ & $\begin{array}{l}\text { Emergence- } \\
\text { harvest }\end{array}$ & $\begin{array}{l}\text { Planting date- } \\
\text { harvest }\end{array}$ \\
\hline \multirow{3}{*}{$\begin{array}{l}\text { Parent } \\
\text { crop }^{1}\end{array}$} & Grande Naine & $14.7 \mathrm{a}$ & $30.8 \mathrm{c}$ & 09/07/98 b & $29 / 11 / 98 c$ & $323 b$ & $146 \mathrm{~b}$ & $467 \mathrm{c}$ \\
\hline & Gruesa & $14.9 \mathrm{a}$ & $33.7 \mathrm{a}$ & $28 / 07 / 98$ a & $27 / 12 / 98$ a & $342 \mathrm{a}$ & $153 \mathrm{a}$ & $495 \mathrm{a}$ \\
\hline & Zelig & $14.8 \mathrm{a}$ & $32.1 \mathrm{~b}$ & $16 / 07 / 98 b$ & $14 / 12 / 98 b$ & $330 \mathrm{~b}$ & $151 \mathrm{ab}$ & $481 \mathrm{~b}$ \\
\hline \multirow{3}{*}{$\begin{array}{l}\text { First } \\
\text { ratoon } \\
\text { crop }^{2}\end{array}$} & Grande Naine & $9.9 \mathrm{~b}$ & $21.4 \mathrm{~b}$ & 03/08/99 b & $09 / 01 / 00 \mathrm{~b}$ & $714 \mathrm{~b}$ & $159 \mathrm{~b}$ & $873 b$ \\
\hline & Gruesa & $10.2 \mathrm{a}$ & $25.0 \mathrm{a}$ & $27 / 08 / 99$ a & $14 / 02 / 00 \mathrm{a}$ & 737 a & $171 \mathrm{a}$ & 908 a \\
\hline & Zelig & $9.7 \mathrm{~b}$ & $21.0 \mathrm{~b}$ & $07 / 08 / 99 \mathrm{~b}$ & $16 / 01 / 00 \mathrm{~b}$ & $717 \mathrm{~b}$ & $163 \mathrm{~b}$ & $880 \mathrm{~b}$ \\
\hline \multirow{3}{*}{$\begin{array}{l}\text { Second } \\
\text { ratoon } \\
\text { crop }^{3}\end{array}$} & Grande Naine & $11.7 \mathrm{ab}$ & $19.7 \mathrm{~b}$ & $23 / 08 / 00 \mathrm{~b}$ & 09/02/01 b & $1099 \mathrm{~b}$ & $170 \mathrm{~b}$ & $1269 \mathrm{~b}$ \\
\hline & Gruesa & $12.1 \mathrm{a}$ & $23.7 \mathrm{a}$ & $10 / 09 / 00 \mathrm{a}$ & 05/03/01 a & $1118 \mathrm{a}$ & $176 a$ & $1293 \mathrm{a}$ \\
\hline & Zelig & $11.4 \mathrm{~b}$ & $20.2 \mathrm{~b}$ & $24 / 08 / 00 \mathrm{~b}$ & $10 / 02 / 01 b$ & $1100 \mathrm{~b}$ & $170 \mathrm{~b}$ & $1270 \mathrm{~b}$ \\
\hline
\end{tabular}

121 Dec. to 21 May; ${ }^{2} 21$ Jan. to 14 June; ${ }^{3} 17$ Feb. to 12 July.

(b) Cultivar comparison in northern trial plots.

\begin{tabular}{|c|c|c|c|c|c|c|c|c|}
\hline \multirow[t]{2}{*}{ Cycle } & \multirow[t]{2}{*}{ Cultivar } & \multicolumn{2}{|c|}{ Leaves emitted $>25 \mathrm{~cm}$} & \multicolumn{2}{|c|}{ Bunch } & \multicolumn{3}{|c|}{ Intervals (days) from planting date } \\
\hline & & $\begin{array}{l}\text { See interval } 1,2 \text { or } 3 \\
\text { according to the cycle }\end{array}$ & $\begin{array}{c}\text { Total at } \\
\text { emergence }\end{array}$ & Emergence & Harvest & $\begin{array}{l}\text { Planting date- } \\
\text { emergence }\end{array}$ & $\begin{array}{l}\text { Emergence- } \\
\text { harvest }\end{array}$ & $\begin{array}{c}\text { Planting date- } \\
\text { harvest }\end{array}$ \\
\hline \multirow{3}{*}{$\begin{array}{l}\text { Parent } \\
\text { crop }{ }^{1}\end{array}$} & Grande Naine & $13.1 \mathrm{a}$ & $31.9 \mathrm{~b}$ & $20 / 08 / 98$ a & $14 / 01 / 99 a$ & $374 \mathrm{a}$ & $167 \mathrm{a}$ & $521 \mathrm{a}$ \\
\hline & Gruesa & $13.4 \mathrm{a}$ & $33.2 \mathrm{a}$ & $23 / 08 / 98 a$ & $22 / 01 / 99$ a & $376 a$ & $173 a$ & $529 a$ \\
\hline & Zelig & $13.1 \mathrm{a}$ & $32.0 \mathrm{~b}$ & $19 / 08 / 98 a$ & $19 / 01 / 99 a$ & $372 a$ & $175 a$ & $526 a$ \\
\hline \multirow{3}{*}{$\begin{array}{l}\text { First } \\
\text { ratoon } \\
\text { crop }^{2}\end{array}$} & Grande Naine & $7.7 \mathrm{a}$ & $20.3 \mathrm{~b}$ & $19 / 09 / 99 b$ & $12 / 04 / 00 \mathrm{a}$ & $769 \mathrm{~b}$ & $211 a$ & 978 a \\
\hline & Gruesa & $8.0 \mathrm{a}$ & $23.0 \mathrm{a}$ & 29/10/99 a & $05 / 05 / 00$ a & 806 a & $200 \mathrm{a}$ & 998 a \\
\hline & Zelig & $8.2 \mathrm{a}$ & $19.7 \mathrm{~b}$ & $10 / 09 / 99 \mathrm{~b}$ & $14 / 04 / 04 a$ & $762 b$ & $218 a$ & $977 \mathrm{a}$ \\
\hline \multirow{3}{*}{$\begin{array}{l}\text { Second } \\
\text { ratoon } \\
\text { crop }^{3}\end{array}$} & Grande Naine & $4.7 \mathrm{a}$ & $17.3 \mathrm{~b}$ & $05 / 10 / 00 a$ & $16 / 04 / 01 \mathrm{a}$ & $1152 \mathrm{a}$ & $192 a$ & $1344 \mathrm{a}$ \\
\hline & Gruesa & $5.8 \mathrm{a}$ & $22.9 \mathrm{a}$ & $25 / 10 / 00 a$ & $14 / 05 / 01 \mathrm{a}$ & $1171 \mathrm{a}$ & $201 a$ & $1372 \mathrm{a}$ \\
\hline & Zelig & $4.4 \mathrm{a}$ & $17.6 \mathrm{~b}$ & $27 / 09 / 00$ a & $22 / 04 / 01$ a & $1143 a$ & $208 a$ & $1351 \mathrm{a}$ \\
\hline
\end{tabular}

110 Dec. to 27 May; ${ }^{2} 11$ Feb. to 10 June; ${ }^{3} 23$ May to 10 Jan.

(c) Comparison of greenhouse covers (PE: 720-gauge polyethylene on all sides and roof; MM: mixed monofilament/raffia mesh on sides and roof; M $6 \times 6$ : 6-by-6 monofilament mesh for roof and monofilament/raffia-mix mesh for sides) in northern trial plots.

\begin{tabular}{|c|c|c|c|c|c|c|c|c|}
\hline \multirow[t]{2}{*}{ Cycle } & \multirow{2}{*}{$\begin{array}{l}\text { Greenhouse } \\
\text { cover }\end{array}$} & \multicolumn{2}{|c|}{ Leaves emitted $>25 \mathrm{~cm}$} & \multicolumn{2}{|c|}{ Bunch } & \multicolumn{3}{|c|}{ Intervals (days) from planting date (PD) } \\
\hline & & $\begin{array}{l}\text { See interval } 1,2 \text { or } 3 \\
\text { according to the cycle }\end{array}$ & $\begin{array}{l}\text { Total at } \\
\text { emergence }\end{array}$ & Emergence & Harvest & $\begin{array}{l}\text { Planting date- } \\
\text { emergence }\end{array}$ & $\begin{array}{l}\text { Emergence- } \\
\text { harvest }\end{array}$ & $\begin{array}{c}\text { Planting date- } \\
\text { harvest }\end{array}$ \\
\hline \multirow{4}{*}{$\begin{array}{l}\text { Parent } \\
\text { crop }^{1}\end{array}$} & PE & $14.2 \mathrm{a}$ & $32.3 b$ & $26 / 07 / 98$ c & $26 / 12 / 98$ c & $346 \mathrm{c}$ & $157 \mathrm{c}$ & $502 \mathrm{c}$ \\
\hline & MM & $12.9 \mathrm{~b}$ & $30.7 \mathrm{c}$ & $31 / 07 / 98$ bc & $19 / 01 / 99 b$ & 354 bc & $173 b$ & $527 \mathrm{~b}$ \\
\hline & $M 6 \times 6$ & $12.5 \mathrm{~b}$ & $31.3 \mathrm{c}$ & 08/08/98 b & 09/02/99 a & $361 \mathrm{~b}$ & $186 a$ & 548 a \\
\hline & Open air ${ }^{4}$ & $12.4 \mathrm{~b}$ & $35.2 \mathrm{a}$ & $19 / 10 / 98$ a & - & $434 \mathrm{a}$ & -- & - \\
\hline \multirow{3}{*}{$\begin{array}{l}\text { First } \\
\text { ratoon } \\
\text { crop }^{2}\end{array}$} & PE & $8.6 \mathrm{a}$ & $22.0 \mathrm{a}$ & $12 / 09 / 99 \mathrm{~b}$ & $03 / 04 / 00 \mathrm{a}$ & $756 \mathrm{~b}$ & $203 a$ & 967 a \\
\hline & MM & $7.6 \mathrm{a}$ & $21.0 \mathrm{ab}$ & $29 / 09 / 99 a b$ & $26 / 04 / 00$ a & $781 \mathrm{ab}$ & $209 a$ & $993 a$ \\
\hline & $M 6 \times 6$ & $7.8 \mathrm{a}$ & $20.1 \mathrm{~b}$ & $18 / 10 / 99$ a & $30 / 04 / 00$ a & $800 \mathrm{a}$ & 218 a & 994 a \\
\hline \multirow{3}{*}{$\begin{array}{l}\text { Second } \\
\text { ratoon } \\
\text { crop }^{3}\end{array}$} & PE & $5.9 \mathrm{a}$ & $18.8 \mathrm{~b}$ & $17 / 09 / 00 \mathrm{~b}$ & $21 / 03 / 01 b$ & $1133 b$ & $185 b$ & $1320 \mathrm{~b}$ \\
\hline & $\mathrm{MM}$ & $5.0 \mathrm{a}$ & $20.4 \mathrm{a}$ & $26 / 10 / 00 \mathrm{a}$ & $17 / 05 / 01$ a & $1172 \mathrm{a}$ & $203 a$ & $1374 \mathrm{a}$ \\
\hline & M $6 \times 6$ & $4.0 \mathrm{a}$ & $18.7 \mathrm{~b}$ & $14 / 10 / 00 a b$ & $15 / 05 / 01 \mathrm{a}$ & $1161 \mathrm{~b}$ & $212 a$ & $1373 a$ \\
\hline
\end{tabular}

110 Dec. to $27 \mathrm{May} ;{ }^{2} 21$ Jan. to 14 June; ${ }^{3} 17$ Feb. to 12 July.

Means followed by the same letters are not significantly different $(p<0.05)$, applying Tukey's test.

${ }^{4}$ Data only available for parent crop cycle (hands), due to storm damage. 
Table IV.

Production characteristics of banana cvs. Grande Naine, Gruesa and Zelig, grown in the south and north of Tenerife (Canary Islands, Spain).

(a) Cultivar comparison in southern trial plots.

\begin{tabular}{|c|c|c|c|c|c|c|c|c|c|c|}
\hline \multirow[t]{3}{*}{ Cycle } & \multirow[t]{3}{*}{ Cultivar } & \multirow{2}{*}{\multicolumn{2}{|c|}{ Bunch }} & \multicolumn{3}{|c|}{ 2nd superior hand } & \multicolumn{3}{|c|}{ 2nd inferior hand } & \multirow{3}{*}{$\begin{array}{c}\text { Net yield } \\
\left(\mathrm{kg}^{-h^{-1}} \cdot \text { year }^{-1}\right)\end{array}$} \\
\hline & & & & \multirow{2}{*}{$\begin{array}{l}\text { No. } \\
\text { fingers }\end{array}$} & \multicolumn{2}{|c|}{ Characteristic finger } & \multirow{2}{*}{$\begin{array}{l}\text { No. } \\
\text { fingers }\end{array}$} & \multicolumn{2}{|c|}{ Characteristic finger } & \\
\hline & & Hands & $\begin{array}{l}\text { Weight } \\
(\mathrm{kg})\end{array}$ & & $\begin{array}{l}\text { Length } \\
(\mathrm{cm})\end{array}$ & $\begin{array}{l}\text { Width } \\
(\mathrm{mm})\end{array}$ & & $\begin{array}{l}\text { Length } \\
(\mathrm{cm})\end{array}$ & $\begin{array}{l}\text { Width } \\
(\mathrm{mm})\end{array}$ & \\
\hline $\begin{array}{l}\text { Parent } \\
\text { crop }\end{array}$ & $\begin{array}{c}\text { Grande Naine } \\
\text { Gruesa } \\
\text { Zelig }\end{array}$ & $\begin{array}{l}11.9 \mathrm{c} \\
13.6 \mathrm{a} \\
12.5 \mathrm{~b}\end{array}$ & $\begin{array}{l}51.2 \mathrm{a} \\
50.6 \mathrm{a} \\
50.2 \mathrm{a}\end{array}$ & $\begin{array}{l}- \\
- \\
-\end{array}$ & $\begin{array}{l}24.5 \mathrm{a} \\
21.5 \mathrm{~b} \\
24.0 \mathrm{a}\end{array}$ & $\begin{array}{l}38.7 \mathrm{a} \\
36.9 \mathrm{c} \\
37.9 \mathrm{~b}\end{array}$ & $\begin{array}{l}- \\
- \\
-\end{array}$ & $\begin{array}{l}21.3 \mathrm{a} \\
19.4 \mathrm{~b} \\
21 . \mathrm{a}\end{array}$ & $\begin{array}{l}37.6 \mathrm{a} \\
35.9 \mathrm{c} \\
36.9 \mathrm{~b}\end{array}$ & $\begin{array}{l}82183 \\
76598 \\
78103\end{array}$ \\
\hline $\begin{array}{l}\text { First } \\
\text { ratoon } \\
\text { crop }\end{array}$ & $\begin{array}{c}\text { Grande Naine } \\
\text { Gruesa } \\
\text { Zelig }\end{array}$ & $\begin{array}{l}14.0 \mathrm{~b} \\
14.8 \mathrm{a} \\
13.8 \mathrm{~b}\end{array}$ & $\begin{array}{l}50.9 a \\
49.5 a \\
48.6 a\end{array}$ & $\begin{array}{l}23.9 \mathrm{~b} \\
26.1 \mathrm{a} \\
24.3 \mathrm{~b}\end{array}$ & $\begin{array}{l}24.4 \mathrm{a} \\
22.5 \mathrm{c} \\
23.9 \mathrm{~b}\end{array}$ & $\begin{array}{l}35.5 \mathrm{a} \\
35.1 \mathrm{a} \\
35.2 \mathrm{a}\end{array}$ & $\begin{array}{c}16.6 \mathrm{~b} \\
17.1 \mathrm{a} \\
16.9 \mathrm{ab}\end{array}$ & $\begin{array}{l}20.0 \mathrm{a} \\
18.8 \mathrm{c} \\
19.4 \mathrm{~b}\end{array}$ & $\begin{array}{l}34.5 \mathrm{a} \\
33.6 \mathrm{~b} \\
34.2 \mathrm{a}\end{array}$ & $\begin{array}{c}104513 \\
101655 \\
99684\end{array}$ \\
\hline $\begin{array}{l}\text { Second } \\
\text { ratoon } \\
\text { crop }\end{array}$ & $\begin{array}{c}\text { Grande Naine } \\
\text { Gruesa } \\
\text { Zelig }\end{array}$ & $\begin{array}{l}13.0 \mathrm{~b} \\
14.3 \mathrm{a} \\
13.0 \mathrm{~b}\end{array}$ & $\begin{array}{l}48.6 \mathrm{a} \\
48.3 \mathrm{a} \\
44.2 \mathrm{~b}\end{array}$ & $\begin{array}{l}24.4 \mathrm{~b} \\
25.5 \mathrm{a} \\
23.2 \mathrm{c}\end{array}$ & $\begin{array}{l}25 a \\
22.5 c \\
24.4 b\end{array}$ & $\begin{array}{c}35.7 \mathrm{a} \\
34.9 \mathrm{~b} \\
35.5 \mathrm{ab}\end{array}$ & $\begin{array}{l}16.5 \mathrm{~b} \\
17.4 \mathrm{a} \\
16.8 \mathrm{~b}\end{array}$ & $\begin{array}{l}20.6 \mathrm{a} \\
19.0 \mathrm{c} \\
19.9 \mathrm{~b}\end{array}$ & $\begin{array}{c}34.6 \mathrm{a} \\
33.8 \mathrm{~b} \\
34.1 \mathrm{ab}\end{array}$ & $\begin{array}{l}99693 \\
99203 \\
90716\end{array}$ \\
\hline \multicolumn{11}{|c|}{ (b) Cultivar comparison in northern trial plots. } \\
\hline \multirow[t]{3}{*}{ Cycle } & \multirow[t]{3}{*}{ Cultivar } & \multirow{2}{*}{\multicolumn{2}{|c|}{ Bunch }} & \multicolumn{3}{|c|}{ 2nd superior hand } & \multicolumn{3}{|c|}{ 2nd inferior hand } & Net yield \\
\hline & & & & \multirow{2}{*}{$\begin{array}{l}\text { No. } \\
\text { fingers }\end{array}$} & \multicolumn{2}{|c|}{ Characteristic finger } & \multirow{2}{*}{$\begin{array}{l}\text { No. } \\
\text { fingers }\end{array}$} & \multicolumn{2}{|c|}{ Characteristic finger } & \\
\hline & & Hands & $\begin{array}{l}\text { Weight } \\
(\mathrm{kg})\end{array}$ & & $\begin{array}{l}\text { Length } \\
(\mathrm{cm})\end{array}$ & $\begin{array}{l}\text { Width } \\
(\mathrm{mm})\end{array}$ & & $\begin{array}{l}\text { Length } \\
(\mathrm{cm})\end{array}$ & $\begin{array}{l}\text { Width } \\
(\mathrm{mm})\end{array}$ & \\
\hline $\begin{array}{l}\text { Parent } \\
\text { crop }\end{array}$ & $\begin{array}{c}\text { Grande Naine } \\
\text { Gruesa } \\
\text { Zelig }\end{array}$ & $\begin{array}{l}11.6 \mathrm{~b} \\
12.7 \mathrm{a} \\
11.9 \mathrm{~b}\end{array}$ & $\begin{array}{l}47.2 \mathrm{a} \\
45.2 \mathrm{~b} \\
44.9 \mathrm{~b}\end{array}$ & $\begin{array}{l}- \\
- \\
-\end{array}$ & $\begin{array}{l}24.6 \mathrm{a} \\
21.6 \mathrm{~b} \\
24.4 \mathrm{a}\end{array}$ & $\begin{array}{l}37.2 \mathrm{a} \\
36.9 \mathrm{a} \\
36.8 \mathrm{a}\end{array}$ & $\begin{array}{l}- \\
- \\
-\end{array}$ & $\begin{array}{l}20.7 \mathrm{a} \\
18.3 \mathrm{~b} \\
20.6 \mathrm{a}\end{array}$ & $\begin{array}{l}35.5 \mathrm{a} \\
33.8 \mathrm{~b} \\
35.3 \mathrm{a}\end{array}$ & $\begin{array}{l}68119 \\
64109 \\
64146\end{array}$ \\
\hline $\begin{array}{l}\text { First } \\
\text { ratoon } \\
\text { crop }\end{array}$ & $\begin{array}{c}\text { Grande Naine } \\
\text { Gruesa } \\
\text { Zelig }\end{array}$ & $\begin{array}{l}12.7 \mathrm{a} \\
12.1 \mathrm{a} \\
12.2 \mathrm{a}\end{array}$ & $\begin{array}{l}44.4 \mathrm{a} \\
36.4 \mathrm{~b} \\
39.0 \mathrm{~b}\end{array}$ & $\begin{array}{l}23.5 \mathrm{a} \\
24.2 \mathrm{a} \\
22.8 \mathrm{a}\end{array}$ & $\begin{array}{l}25.3 \mathrm{a} \\
22.9 \mathrm{c} \\
24.0 \mathrm{~b}\end{array}$ & $\begin{array}{l}36.0 \mathrm{a} \\
36.0 \mathrm{a} \\
35.0 \mathrm{a}\end{array}$ & $\begin{array}{l}16.8 \mathrm{a} \\
17.2 \mathrm{a} \\
17.0 \mathrm{a}\end{array}$ & $\begin{array}{l}20.1 \mathrm{a} \\
18.4 \mathrm{~b} \\
20.0 \mathrm{a}\end{array}$ & $\begin{array}{l}34.0 \mathrm{a} \\
33.0 \mathrm{~b} \\
33.0 \mathrm{~b}\end{array}$ & $\begin{array}{l}69277 \\
55223 \\
61396\end{array}$ \\
\hline $\begin{array}{l}\text { Second } \\
\text { ratoon } \\
\text { crop }\end{array}$ & $\begin{array}{c}\text { Grande Naine } \\
\text { Gruesa } \\
\text { Zelig }\end{array}$ & $\begin{array}{l}12.5 \mathrm{a} \\
12.8 \mathrm{a} \\
12.5 \mathrm{a}\end{array}$ & $\begin{array}{l}45.7 \mathrm{a} \\
47.4 \mathrm{a} \\
45.8 \mathrm{a}\end{array}$ & $\begin{array}{l}23.6 \mathrm{a} \\
23.6 \mathrm{a} \\
22.8 \mathrm{a}\end{array}$ & $\begin{array}{l}26.0 \mathrm{a} \\
24.1 \mathrm{~b} \\
25.1 \mathrm{ab}\end{array}$ & $\begin{array}{l}36.0 \mathrm{a} \\
38.0 \mathrm{a} \\
36.0 \mathrm{a}\end{array}$ & $\begin{array}{l}16.6 \mathrm{a} \\
16.8 \mathrm{a} \\
16.8 \mathrm{a}\end{array}$ & $\begin{array}{l}20.2 \mathrm{a} \\
18.5 \mathrm{c} \\
19.4 \mathrm{~b}\end{array}$ & $\begin{array}{c}34.0 \mathrm{a} \\
35.0 \mathrm{~b} \\
34.0 \mathrm{ab}\end{array}$ & $\begin{array}{l}78310 \\
80038 \\
77196\end{array}$ \\
\hline
\end{tabular}

(c) Comparison of greenhouse covers (PE: 720-gauge polyethylene on all sides and roof; MM: mixed monofilament / raffia mesh on sides and roof; M $6 \times 6$ : 6-by- 6 monofilament mesh for roof and monofilament/raffia-mix mesh for sides) in northern trial plots.

\begin{tabular}{|c|c|c|c|c|c|c|c|c|c|c|}
\hline \multirow[t]{3}{*}{ Cycle } & \multirow{3}{*}{$\begin{array}{c}\text { Greenhouse } \\
\text { cover }\end{array}$} & \multirow{2}{*}{\multicolumn{2}{|c|}{ Bunch }} & \multicolumn{3}{|c|}{ 2nd superior hand } & \multicolumn{3}{|c|}{ 2nd inferior hand } & \multirow{3}{*}{ 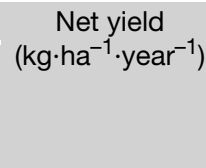 } \\
\hline & & & & \multirow{2}{*}{$\begin{array}{l}\text { No. } \\
\text { fingers }\end{array}$} & \multicolumn{2}{|c|}{ Characteristic finger } & \multirow{2}{*}{$\begin{array}{l}\text { No. } \\
\text { fingers }\end{array}$} & \multicolumn{2}{|c|}{ Characteristic finger } & \\
\hline & & Hands & $\begin{array}{l}\text { Weight } \\
(\mathrm{kg})\end{array}$ & & $\begin{array}{l}\text { Length } \\
(\mathrm{cm})\end{array}$ & $\begin{array}{l}\text { Width } \\
(\mathrm{mm})\end{array}$ & & $\begin{array}{l}\text { Length } \\
(\mathrm{cm})\end{array}$ & $\begin{array}{l}\text { Width } \\
(\mathrm{mm})\end{array}$ & \\
\hline $\begin{array}{l}\text { Parent } \\
\text { crop }\end{array}$ & $\begin{array}{c}\text { PE } \\
\text { MM } \\
\text { M6 } 66 \\
\text { Open-air }{ }^{1}\end{array}$ & $\begin{array}{l}12.9 a \\
12.6 \mathrm{a} \\
12.5 \mathrm{a} \\
10.3 \mathrm{~b}\end{array}$ & $\begin{array}{c}48.5 \mathrm{a} \\
44.3 \mathrm{~b} \\
44.6 \mathrm{~b} \\
-\end{array}$ & $\begin{array}{l}- \\
- \\
- \\
-\end{array}$ & $\begin{array}{c}23.8 \mathrm{a} \\
24.0 \mathrm{a} \\
22.9 \mathrm{~b} \\
-\end{array}$ & $\begin{array}{c}37.5 \mathrm{a} \\
37.3 \mathrm{a} \\
36.1 \mathrm{~b} \\
-\end{array}$ & $\begin{array}{l}- \\
- \\
-\end{array}$ & $\begin{array}{c}19.8 \mathrm{a} \\
19.9 \mathrm{a} \\
19.9 \mathrm{a} \\
-\end{array}$ & $\begin{array}{c}34.5 \mathrm{a} \\
35.1 \mathrm{a} \\
35.0 \mathrm{a} \\
-\end{array}$ & $\begin{array}{c}72291 \\
63055 \\
61029 \\
-\end{array}$ \\
\hline $\begin{array}{l}\text { First } \\
\text { ratoon } \\
\text { crop }\end{array}$ & $\begin{array}{c}\text { PE } \\
\text { MM } \\
M 6 \times 6\end{array}$ & $\begin{array}{l}12.5 \mathrm{a} \\
12.3 \mathrm{a} \\
12.2 \mathrm{a}\end{array}$ & $\begin{array}{l}41.7 \mathrm{a} \\
36.8 \mathrm{~b} \\
41.2 \mathrm{a}\end{array}$ & $\begin{array}{l}23.5 a \\
22.8 a \\
23.6 a\end{array}$ & $\begin{array}{c}24.6 \mathrm{a} \\
24.1 \mathrm{ab} \\
23.6 \mathrm{~b}\end{array}$ & $\begin{array}{l}36.0 \mathrm{a} \\
36.0 \mathrm{a} \\
35.0 \mathrm{a}\end{array}$ & $\begin{array}{l}16.9 a \\
16.8 \mathrm{a} \\
17.3 \mathrm{a}\end{array}$ & $\begin{array}{l}19.4 \mathrm{a} \\
19.3 \mathrm{a} \\
19.8 \mathrm{a}\end{array}$ & $\begin{array}{l}33.0 \mathrm{a} \\
34.0 \mathrm{a} \\
33.0 \mathrm{a}\end{array}$ & $\begin{array}{l}63992 \\
56253 \\
65651\end{array}$ \\
\hline $\begin{array}{l}\text { Second } \\
\text { ratoon } \\
\text { crop }\end{array}$ & $\begin{array}{c}\mathrm{PE} \\
\mathrm{MM} \\
\mathrm{M} 6 \times 6\end{array}$ & $\begin{array}{l}12.7 \mathrm{a} \\
12.5 \mathrm{a} \\
12.6 \mathrm{a}\end{array}$ & $\begin{array}{l}46.2 \mathrm{a} \\
48.0 \mathrm{a} \\
44.7 \mathrm{a}\end{array}$ & $\begin{array}{l}22.9 \mathrm{a} \\
23.5 \mathrm{a} \\
23.5 \mathrm{a}\end{array}$ & $\begin{array}{l}25.7 \mathrm{a} \\
25.6 \mathrm{a} \\
23.8 \mathrm{~b}\end{array}$ & $\begin{array}{l}37.0 \mathrm{a} \\
38.0 \mathrm{a} \\
36.0 \mathrm{a}\end{array}$ & $\begin{array}{l}16.6 \mathrm{a} \\
16.9 \mathrm{a} \\
16.7 \mathrm{a}\end{array}$ & $\begin{array}{l}19.6 \mathrm{a} \\
19.7 \mathrm{a} \\
18.8 \mathrm{~b}\end{array}$ & $\begin{array}{l}34.0 \mathrm{a} \\
35.0 \mathrm{a} \\
34.0 \mathrm{a}\end{array}$ & $\begin{array}{l}82785 \\
78724 \\
74343\end{array}$ \\
\hline
\end{tabular}


The number of hands per bunch was significantly greatest for Gruesa in all cycles in the southern trial but only for the first cycle in the northern locality. During the first cycle of the southern trial, Zelig and Grande Naine differed significantly, in favour of Zelig. Significant differences between Zelig and Grande Naine, in favour of the former, were found for the first cycle of the southern trial but levelled off in successive cycles; no differences were observed in the northern trial. In the northern trial (first cycle only), the differences were significant, clearly in favour of greenhouse versus open air; no differences were observed between the three greenhouse plots.

For the characteristics of fruits of the superior and inferior second hands (length and width), Grande Naine exhibited significantly the longest fruits in all treatments (southern and northern trials), followed first by Zelig and finally by Gruesa; the fingers of the second superior hand had greater length in the PE and MM plots compared with the M $6 \times 6$ one; this was also true for the inferior hand but it was only significant in the third cycle. Widths of first-cycle fruits were also significantly different for both hands in the southern trial, following the same Grande Naine > Zelig > Gruesa pattern established for length, although differences were much reduced in successive cycles. In the northern trial, widths of the fingers of the inferior hand were significantly different in the first cycle, favourable to both Grande Naine and Zelig compared with Gruesa, and, in the second cycle, they were favourable to Grande Naine compared with Gruesa and Zelig. The greenhouse characteristic fruit widths were significantly higher in the PE and MM plots than in the M $6 \times 6$ one, but this was true only for superior second hands in the first cycle, although no significant differences were detected for inferior second hands in any cycle.

Significant differences regarding the number of fruits of the superior and inferior second hands were detected in the southern trial in favour of Gruesa versus Grande Naine and Zelig in the second cycle and between the three Gruesa > Grande Naine > Zelig in the third cycle.

\subsection{Notes on greenhouse covers}

The spectroradiometric measurement (figure 2) show clear differences between the three covers, particularly in their capacity to limit UV penetration: $\mathrm{PE}$ allowed 28\%, MM $69 \%$ and M $6 \times 684 \%$. The highest temperatures (figure 3) were reached under the PE cover although MM registered closely behind; M $6 \times 6$ was notably inferior, virtually equating the open-air registers for the northern locality. In any case, observed differences attenuated during the shorter autumn-winter days.

Whitefly is prevalent in the north of Tenerife and the PE cladding, compared with the other two types, clearly reduced the incidence of attacks by both Aleurodicus dispersus and Leucanoides floccissimus, two important pests of the banana and other crops in the Canary Islands.

\section{Discussion}

\subsection{Morphological characteristics}

The pseudostem values found for Grande Naine and Zelig coincide generally with those reported in previous works in the Canaries and elsewhere. The cultivar Gruesa has never been evaluated before but, in view of the data generated by the present trials, Gruesa has a clearly lower [height:width] ratio than that reported for other Dwarf Cavendish selections [2, 4-6] which, together with its thickness, explains why local growers began referring to it as "Gruesa" ("thick" in Spanish). Additionally, as evidenced after the 1999 storms (data not shown), the greater width and small stature of Gruesa give it a clear advantage over the other cultivars, which could be of great interest not only for the Canary Islands but also for other banana areas of the world.

As in previous trials [2], the pseudostem [height:width] ratio seems to be fully linked to cultivars independent of environmental conditions, which indicates the validity of this criterion for cultivar discrimination [2], as it remains stable, independent of location 
and even under different cropping systems (open-air or greenhouse). Height alone should not be used as a distinguishing parameter: while it does vary between cultivars, in any given cultivar it is susceptible to appreciable changes in function of the climate or microclimate (e.g., greenhouse) of the plantation, as temperature is the main factor (besides water stress) governing growth and development [7].

Thickness, like height, was less influenced by environment, with Gruesa clearly different from Grande Naine and Zelig. Previous results on the greater height and width of greenhouse-cultivated plants are also confirmed compared with open-air plants growing in the same plot [4], doubtless as a consequence of the better climatic conditions provided by greenhouses.

\subsection{Phenological characteristics}

The differences in leaf emission rate in the southern trial, in favour of Gruesa, coincide with the results of previous studies done in the Canary Islands, comparing the traditional Dwarf Cavendish with Grande Naine and Williams (of which Zelig is a mutation) [2, 3], as well as in South Africa [8]; all of which appear to validate the hypothesis that this cultivar, Gruesa, responds better regarding leaf emission rate than others to a relatively cooler, subtropical climate. As pertains to the present study, this hypothesis gains further credibility in view of the results of the greenhouse trials, where no differences were found (they do, however, exist between Gruesa and Grande Naine, although not significantly so).

The cause of the differences seen among greenhouse plots and between these and the open-air plot of the northern locality may be due to multiple factors: temperature, light quality, and greater and/or varying degrees of protection of the leaves against the wind. The differences in total number of leaves emitted until bunch emergence, smaller for Grande Naine and Zelig than for Gruesa, which directly influences cycle length and consequently yield, are doubtless due to intrinsic differences in the cultivars of the Cavendish subgroup which have been amply studied [9-15], although not compared until now.

These reported differences in the subgroup cultivars go some way towards explaining why results for total number of leaves emitted until flowering vary in different cycles in the greenhouse plots. In fact, these differences surpass the advantages conferred by the greater leaf emission rate of Gruesa, leading to a later bunch emergence, due to the greater number of leaves emitted by this last cultivar. Coupling this flowering delay with a longer emergence-harvest interval, and even with a longer planting-harvest interval, can explain not only possible varietal differences but also why the emergence-harvest interval in the subtropical conditions of the Canaries can vary depending on the seasons: the longest being those of autumn and the shortest, those of the summer months [13-15]. In view of this, it is questionable if Gruesa is suitable for the cooler subtropical areas of the Canary Islands, where a longer cycle reduces the probability of a yearly crop, which would in turn have serious effects on grower income. In this sense, local growers who want a one-cycle crop [16] do use only Grande Naine, although such a decision may be influenced by the fact that Grande Naine does not experience the flower obstruction typically associated with Dwarf Cavendish [7] and observed in Gruesa in some localities of the Canary Islands (data not shown).

The higher temperatures recorded in the PE greenhouse structure, compared with the MM and M $6 \times 6$ structures, may be why this treatment had the shortest cycle, as temperature is the main factor governing growth and development of the banana plant [7].

\subsection{Production characteristics}

Our results, in which Grande Naine bunch weight was always superior to Zelig (although not always significantly so), are in line with previous work [4]. Although Zelig does have the advantage of being shorter, it did not show to advantage in any of the other parameters so it is not necessary to continue comparing Grande Naine and Zelig. This is not the case for Gruesa, which is not only 
better able to withstand wind damage but exhibits better morphological characteristics, such as height and width, as well as higher yields, very close, in fact, to those recorded for Grande Naine.

Local growers have been quick to pick up on these qualities and new plantations are based almost exclusively on Gruesa and Grande Naine, even while acknowledging that Gruesa produced somewhat less than Grande Naine earlier [2], its shorter height makes crop management easier.

Work in other countries reported increased bunch weights after the first cycle for cultivars of the Cavendish subgroup [17, 18]. It is interesting to note that this has not occurred in the Canaries [3], which may indicate that, under favourable climatic conditions, using tissue culture plants and with appropriate cultural practices, it is possible to obtain high productions, even in the first cycle, for cultivars of the Cavendish subgroup.

Although the shorter length of Gruesa makes it a particularly attractive cultivar for markets that prefer smaller fruit, such as that of mainland Spain, the overall differences in length between the three cultivars are slight enough that to date all three have proved acceptable to the mainland consumer, the largest consumer of Canary bananas.

Further work needs to be done to determine if the greater number of fruits per hand found in Gruesa in this trial is in reality due to a greater proportion of hands bearing three rows of fruit instead of the normal two rows (data not shown). Gruesa growers in several localities have reported this and, if proved true, it would logically affect cluster formation and hence be a defect.

\subsection{General observations on greenhouse covers}

As remarked earlier, greenhouse cultivation reduces whitefly incidence [19]. The greater protection afforded by the PE cladding could be due to the polyethylene limiting UV penetration, as has been found to occur with whitefly species different from those reported for bananas in the Canary Islands and affecting other crops in different countries [20].

The slight increase in yield observed in the PE treatment confirms previous studies [4], validating this type of cladding for use in the cooler areas of the Canary Islands.

\section{Conclusions}

1. Gruesa appears more recommendable than Zelig and Grande Naine for open-air plantations in windy localities, given its better pseudostem [height:width] ratio. In the Canary Islands, growers in these localities routinely plant in greenhouses that preclude the necessity of cultivar selection based on this criterion.

2. Cycle length is slightly longer in Gruesa compared with Grande Naine and Zelig which, under marginal conditions, such as those of the northern slopes of the Canary Islands, can lead to a late harvest date, with unfavourable prices. Due to this longer cycle, Gruesa is not apt for one-cycle use under marginal subtropical conditions.

3. The yield of Zelig is inferior to those of Grande Naine and Gruesa in several localities. Added to the fact that it does not appear to have other redeeming qualities with which to rival the other cultivars, it is safe to conclude that Zelig should not be recommended in the Canary Islands.

4. Gruesa, with the shortest fruit length, presents qualities looked for in certain markets, such as the Spanish mainland. All three cultivars produce fruit well above the minimum EEC requirements for "extra" qualifications (14 cm length and $27 \mathrm{~mm}$ width) [21]

5. All three cultivars are heavy producers in both localities studied (in terms of $\mathrm{kg} \cdot \mathrm{ha}^{-1} \cdot \mathrm{year}^{-1}$ ), with a slight advantage of Grande Naine over the others.

6. The polyethylene cover provided the best environment for crop development, effectively reducing crop cycle and increasing yield. 


\section{Acknowledgements}

To the Instituto Nacional de Investigación y Tecnologia Agraria y Alimentaria (INIA) of Spain for funding the Project SC97-114 INIA Mejora de la Competitividad del plátano en Canarias (II).

\section{References}

[1] Galán Saúco V., Cabrera Cabrera J., Variedades de plátanos cultivadas en Canarias, Merc. 24 (1999) 8-11.

[2] Galán Saúco V., Cabrera Cabrera J., Hernández Delgado P.M., A comparison of bananas cultivar Dwarf Cavendish, Grande Naine and Williams for the Canary Islands, Fruits 50 (4) (1995) 255-256.

[3] Galán Saúco V., Cabrera Cabrera J., Hernández Delgado P.M., Rodríguez Pastor M.C., Evaluation of medium-height Cavendish banana cultivars under the subtropical conditions of the Canary Islands, Acta Hortic. 490 (1998) 103-113.

[4] Galán Saúco V., Cabrera Cabrera J., Hernández Delgado P.M., Rodríguez Pastor M.C., Comparison of protected and open-air cultivation of Grande Naine and Dwarf Cavendish bananas, Acta Hortic. 490 (1998) 247-259.

[5] Eckstein K., Fraser C., Husselmann J., Temple Murray N., The evaluation of promising new banana cultivars, Acta Hortic. 490 (1998) 57-69.

[6] Méndez Hernández C., A comparison of the parent crop of three cultivars of banana in the open air and under plastic mesh in the North of Tenerife, Acta Hortic. 490 (1998) 97101.

[7] Robinson J., Bananas and plantains, CAB Int., Wallingford, UK, 1996.

[8] Robinson J., Characteristic of three new banana cultivars, Ban. Newsl. 12 (1989) 9-10.

[9] Ziv D., L'influence de l'hiver sur la croissance du bananier, in: Goren N., Mendel K. (Eds.), Proc. XVIII Int. Hortic. Congr., Tel Aviv, Israel, 1970, pp. 1-11.

[10] Lassoudière A., Le bananier et sa culture en Côte d'Ivoire. Première partie. Connaissance de la plante. Interaction avec le milieu écologique, Inst. Rech. Fruits Agrumes, Abidjan, Côte d'Ivoire,1978.

[11] Kuhne F.A., The Dwarf Cavendish Banana, CSFRI, Farming in South Africa C3 Nelspruit, South Africa, 1979.

[12] Galán Saúco V., García Samarín J., Carbonell E., Estudio de la práctica del deshijado y la fenología de la platanera [Musa acuminata Colla (AAA) cv. Pequeña Enana] en la isla de Tenerife. I. Introducción y revisión bibliográfica, Fruits 39 (7-8) (1984) 453-459.

[13] Galán Saúco V., García Samarín J., Carbonell E., Estudio de la práctica del deshijado y la fenología de la platanera [Musa acuminata Colla (AAA) cv. Pequeña Enana] en la isla de Tenerife. II. Estudio base de la práctica del deshijado en Tenerife, Fruits 39 (9) (1984) 541-549.

[14] Galán Saúco V., García Samarín J., Carbonell E., Estudio de la práctica del deshijado y la fenología de la platanera [Musa acuminata Colla (AAA) cv. Pequeña Enana] en la isla de Tenerife. III. Fenología de la platanera en la isla de Tenerife, Fruits 39 (10) (1984) 595605.

[15] Galán Saúco V., García Samarín J., Carbonell E., Estudio de la práctica del deshijado y la fenología de la platanera [Musa acuminata Colla (AAA) cv. Pequeña Enana] en la isla de Tenerife. IV. Práctica tentativa deseable para el deshijado en la isla de Tenerife en base a las observaciones fenológicas y discusión general sobre el deshijado en Canarias, Fruits 39 (10) (1984) 606-611.

[16] Cabrera Cabrera, J., Galán Saúco V., Hernández Delgado P.M., Rodríguez Pastor M.C., Single-cycle cultivation of in vitropropagated Grande Naine banana under subtropical conditions, Acta Hortic. 490 (1998) 175-179.

[17] Turner D.W., Hunt N., Growth, yield and leaf nutrient composition of 30 banana varieties in subtropical New South Wales, Techn. Bull. 31, Dep. Agric., New South Wales, Australia, 1984.

[18] Robinson, J.C., Nel D.J., Eckstein K., A field comparison of ten Cavendish subgroup banana cultivars and selections (Musa AAA) over four crop cycles in the subtropics, J. Hortic. Sci. 69 (4) (1993) 511-521.

[19] Hernández Suárez E., Carnero Hernández A., Febles González J.C., Brito Herrera P., Medina Alonso G., Suárez Báez J.M., Amador Martín S., Situación actual de las moscas 
blancas espirales en Canarias, in: Fernández Galván D., Hernández Delgado P.M. (Eds.), Actividades del ICIA en platanera, ICIA, La Laguna, Tenerife, Spain, 2002.

[20] Antignus Y., Nestel D., Cohen, S., Lapidot M., Ultraviolet-deficient greenhouse environmental effects white fly attraction and flight- behavior, Environ. Entomol. 30 (2001) 394399.

[21] Anon., Council Regulation (EEC) No 404/93 of 13 February 1993 on the common organization of the market in bananas, Off. J. Eur. Union, L 047, Brussels, Belgium, 1993, pp. 1-11.

\section{Evaluación comparativa de los cultivares Zelig, Gran Enana y Gruesa bajo distintas condiciones ambientales en las Islas Canarias.}

Resumen - Introducción. Se realizó un estudio para comparar el cultivar Gruesa, cultivar local de Pequeña Enana, con otros dos cultivares del subgrupo Cavendish al aire libre y bajo invernadero en dos localidades de las Islas Canarias. Material y métodos. Los ensayos se realizaron con los cultivares Zelig, Gran Enana y Gruesa en cuatro parcelas de la vertiente norte de la isla de Tenerife: una al aire libre y tres bajo cultivo protegido (una con lámina de polietileno y dos con mallas de diferentes características), así como en una parcela al aire libre en la vertiente sur. Se han estudiado las características morfológicas, fenológicas y productivas durante tres ciclos productivos. Resultados. Se observan diferencias significativas en la altura de las plantas de los distintos cultivares (Gran Enana > Zelig > Gruesa) y grosor del seudotallo (Gruesa $>$ Gran Enana $=$ Zelig). Cada cultivar mantiene una relación [altura:grosor] estable para las distintas condiciones de cultivo. El ciclo productivo del cultivar Gruesa presenta un ligero retraso, precisando emitir más hojas hasta la emergencia del racimo que los otros dos cultivares estudiados. El cultivar Gruesa presenta un mayor número de manos por racimo coincidiendo con un mayor número de dedos por mano. La longitud de los dedos guarda una relación directa con la altura del seudotallo de los tres cultivares. Los tres cultivares muestran una alta productividad, manteniendo unas características organolépticas muy similares. En el caso de la cubierta de polietileno al norte de la isla se observa una reducción en la duración del ciclo, así como un aumento de la productividad frente a las restantes cubiertas y la parcela al aire libre. Conclusión. El cultivar Gruesa parece más recomendable que Zelig y Gran Enana para emplazamientos ventosos al aire libre.

España / Canarias / Musa / ensayos de variedades / sistemas de cultivo / invernaderos / radiación solar / fenología 